Quantum modeling to determine the carcinogenic potential of aflatoxin B1 produced by Aspegillus sp and its metabolic derivate aflatoxin M1

\title{
Modelamiento cuántico para determinar el potencial cancerígeno de la aflatoxina B1 producida por Aspegillus sp y de su derivado metabólico aflatoxina M1
}

Manuel González-Pérez*

División Posgrado en Ciencias de la Ingeniería Biomédica. Universidad Popular Autónoma del Estado de Puebla (UPAEP). Calle 17 sur 901 colonia Santiago (72410) Puebla, Puebla, México.

*Corresponding author.

E-mail address: manuel.gonzalez@upaep.mx (M. González-Pérez).

Article history:

Received: 16 June 2017 / Received in revised form: 27 June 2017 / Accepted: 30 June 2017 / Published online: 1 July 2017

https://doi.org/10.29267/mxjb.2017.2.2.255

\begin{abstract}
Aflatoxin B1 (AFB1) produced mainly by Aspergillus flavors, Aspergillus parasitic and Aspergillus nonius is a potent human and animal hepatocarcinogen. The main objective of this paper was to calculate the AFB1 and aflatoxin M1 (AFM1) Electron Transfer Coefficients (ETC), and the base pairs allowed for both DNA and RNA. We used the Hyperchem quantum simulator for Windows, specifically the PM3 semiempirical method (SE-PM3). The quantum modeling showed that AFB1 toxin $(\mathrm{ETC}=40,533)$ has very high mutagenic potential, but its metabolic derivate AFM1 $(\mathrm{ETC}=36.023)$, which is more soluble because its ETC is lower, has an even greater mutagenic capacity. This means that the human body when trying to eliminate the toxin (AFB1), metabolically transforms it to a substance with greater mutagenic potential (AFM1) and consequently highly carcinogenic. These analyzes confirm the findings found in laboratory experiments in which it is shown that AFB1 is one of the most carcinogenic substances, especially because being metabolized becomes an even more toxic substance for humans. These substances together (AFB1 and AFM1) cause severe mutations to both DNA and RNA. The base pair most likely to bind by AFM1 is C: G; instead the base pair to which AFB1 binds is A: T. Therefore, both have DNA as main target.
\end{abstract}

Keywords: Aflatoxin B1, Aflatoxin M1, Electron Transfer Coefficient, Semiempirical Parametric Method 3. 


\section{RESUMEN}

La aflatoxina B1 (AFB1) es producida principalmente por los hongos Aspergillus flavus, Aspergillus parasiticus y Aspergillus nonius. La AFB1 es un potente hepatocarcinógeno humano y animal. El objetivo principal de este artículo fue calcular los Coeficientes de Transferencia de Electrones (ETC) de la AFB1, aflatoxina M1 (AFM1) y los pares de bases permitidos tanto para ADN como para ARN. Se utilizó el simulador cuántico Hyperchem para Windows, específicamente el método Semiempírico PM3 (SE-PM3). El modelamiento cuántico mostró que la toxina AFB1 (ETC = 40.533) tiene un muy alto potencial mutagénico, pero su derivado metabólico AFM1 (ETC $=36.023)$, que es más soluble dado que su ETC es menor, presenta una capacidad mutagénica aún mayor. Esto quiere decir que el cuerpo humano al tratar de eliminar la toxina (AFB1), la transforma metabólicamente a una sustancia con mayor potential mutagénico (AFM1) y consecuentemente altamente cancerígena. Estos análisis confirman los hallazgos encontrados en experimentos de laboratorio en los que se muestra que la AFB1 es una de las sustancias más cancerígenas que existen, sobretodo porque al ser metabolizada se transforma en una sustancia aún más toxica para el humano. Estas sustancias en conjunto (AFB1 y AFM1) causan severas mutaciones tanto al ADN como al ARN. El par de bases más susceptible de unirse a la AFM1 es C:G; en cambio el par de bases al que se une la AFB1 es A:T. Por lo tanto, ambos tienen como blanco principal al ADN.

Palabras clave: Aflatoxina B1, Aflatoxina M1, Coeficiente de Transferencia de Electrones, Método Semiempirico Paramétrico 3.

\section{INTRODUCCIÓN}

\subsection{Generalidades sobre las aflatoxinas}

Las aflatoxinas son micotoxinas que pueden contaminar cereales, semillas, nueces y frutos deshidratados, según las condiciones de almacenamiento. En particular, la AFB1 producida principalmente por Aspergillus flavus, Aspergillus parasiticus y Aspergillus nonius (Kim et al., 2017), es un potente hepatocarcinógeno humano y animal (Livingstone et al., 2017). La exposición a esta toxina aumenta el riesgo de cirrosis y carcinoma hepatocelular en portadores crónicos de virus de la hepatitis B (Chu et al., 2017). Se están utilizando métodos rápidos y baratos para detectar sus biomarcadores urinarios (Schwartzbord et al., 2017). Entre estos métodos se encuentra la tecnología de secuenciación de consenso dúplex en ratones para identificar tumores cancerígenos hepáticos inducidos por esta toxina ( Fedeles et al., 2017). Otros investigadores utilizan la secuenciación de ADN de alta fidelidad en ratones para revelar espectros mutacionales del carcinógeno hepático causado por la AFB1 (Chawanthayatham et al., 2017).

La exposición aguda a aflatoxinas puede causar la muerte y la enfermedad (aflatoxicosis) en seres humanos. Se ha documentado que las tasas de fatalidad por aflatoxicosis son tan altas como el 40\% en Kenia (Awuor et al., 2017).

En México se han llevado a cabo investigaciones de aflatoxinas (AF) muy interesantes. Una encuesta de tortilla de maíz en México reveló que el 17\% están contaminados con AF. El 
proceso inicial para preparar tortillas incluye hervir los granos de maíz con cal y suponer que se destruyen las AF en tortillas de maíz, protegiendo a los consumidores de sus efectos mutagénicos. En especial, se investigó la mutagenicidad de la AFB1 en tortillas durante la digestión preparando tortillas con pasta contaminada deliberadamente con AFB1 y se demostró que tenían un efecto mutagénico en la prueba de Ames; pero este efecto no se observó en las tortillas preparadas con maíz contaminado naturalmente por FA. El pH alcalino (11.6 - 12.0) del tratamiento de la cal de las tortillas inhibe el efecto mutagénico de la AFB1, mientras que el pH neutro tanto de la saliva (7.0) como del líquido pancreático (7.5) reactiva la mutagenicidad de AFB1 (Moctezuma-Zarate et al., 2017).

Algunos investigadores evaluaron mezclas de toxinas tales como la interacción de AFB1FB1 (fumnonisina B1) para inducir toxicidades genéticas y de ciclo celular en hepatocitos de rata BRL-3A, afectación de especies reactivas de oxígeno (ROS) y las vías de metabolización AFB1 citocromo P450 (CYP) y ácido araquidónico (ArAc) que define el metabolismo como contribuyentes ROS. Se concluyó que la modulación de las vías metabólicas por las mezclas de AFB1-FB1 podría aumentar sus propiedades hepatocarcinógenas (Mary et al., 2017).

Se han hecho esfuerzos por contrarrestar el efecto que produce la ingesta de la toxina AFB1 y se encontró que los polifenoles del te fermentado pueden proteger el daño que produce la AFB1 en el hígado de ratas y se propone como aditivo alimentario (Olsen et al., 2017). Por otro lado se ha propuesto y evaluado la eficacia, aceptabilidad y palatabilidad de la arcilla de montmorillonita cálcica utilizada para reducir la exposición a la dieta de AFB1 en un estudio cruzado en Kenia entre otros esfuerzos (Awuor et al., 2017).

Profundizando las investigaciones de esta toxina cancerígena se determinó la reactividad y la accesibilidad de todos los posibles sitios potenciales del metabolismo de la AFB1. En este sentido, se emplearon cálculos cuánticos según la teoría del funcional de la densidad (DFT, siglas en inglés) para calcular energías de activación para cada reacción posible y simulaciones de dinámica molecular para elucidar cada sitio potencial del metabolismo. Se encontró que el metabolismo de la AFB1 está mediado por citocromo P450 1A2 y 3A4 en las contribuciones de ligandos y proteínas. Los resultados coinciden con los datos experimentales previamente reportados (Lu et al., 2017).

En cuanto a uno de los derivado metabólicos de esta toxina, se encontró que los hábitos alimenticios con más tendencia a consumir pan, arroz y bebidas alcohólicas de cerveza aumentaron significativamente $(\mathrm{P} \leq 0.05)$ el riesgo de aparición de AFM1 en la leche materna. Además, un mayor consumo de pan, oliva y crema tradicional aumentó significativamente $(\mathrm{P} \leq 0.05)$ los niveles de AFM1 en las muestras de leche materna. Los autores piden a la comunidad científica que se deben realizar más investigaciones para determinar con mayor precisión la asociación entre la aparición de AFM1 y factores dietéticos y también el riesgo de exposición infantil a esta micotoxina (Jafari et al., 2017).

Estudios realizados en Serbia durante 2015 mostraron que el nivel más alto de AFM1 en la leche cruda se encontró durante la temporada de otoño, mientras que fue significativamente menor durante el resto del año (Miocinovic et al., 2017). En Croacia se recogieron muestras de leche cruda de vaca, cabra y oveja en diferentes regiones durante la primavera y el otoño 
de 2016 y se encontró que los niveles de AFM1 fueron superiores a los niveles máximos de residuos de la Unión Europea (50 ng/kg) (Bilandžić et al., 2017).

\subsection{Bases nitrogenadas}

Las bases nitrogenadas son la parte medular estructural del ADN y el ARN. Algunas investigaciones hechas con simulación química cuántica empleando métodos semiempíricos como el SE-PM3 arrojan resultados interesantes. Por ejemplo, los pares de bases nitrogenadas permitidas no llevan una secuencia numérica, es decir, no son lineales, ni muestran otro patrón de lugar geométrico en cuanto al valor del Coeficiente de Transferencia de Electrones (ETC) (González-Pérez et al., 2016, 2017). Por esa razón solamente se puede aproximar una probabilidad de interacciones.

El objetivo de este artículo fue calcular los ETCs de la AFB1, AFM1 y los pares permitidos tanto para ADN como para ARN. Con estos cálculos se compararon con bandas cruzadas y se hallaron los pozos cuánticos de energía mínima y se pudo predecir así las interacciones mutagénicas más probables.

\section{MATERIALES Y METODOS}

\subsection{Parámetros generales de la simulación cuántica}

Se utilizó el simulador cuántico Hyperchem para Windows número de serie \#12-8001501800080, fabricado por la empresa Hypercube y distribuido en México por MultiOn, S.A de C.V. Específicamente el método SE-PM3. La geometría de las moléculas se optimizó por el método Polak Ribiere.

Los parámetros específicos de optimización geométrica fueron: Charge and spin. Total Charge 0. Spin Multiplicity 1. SCF Control. Converge Limit 0.01. Interaction Limit 1000. Acelerate converge $=$ Yes. Spin Pairing Lowest. Overlap Weighting Factor. Sigma-Sigma 1. Pi-Pi 1. Polarizabilities do not calculate.

\subsection{Parámetros específicos de la simulación cuántica para la realización de los cálculos}

Parámetros para el cálculo de orbitales moleculares HOMO y LUMO.

Orbitals. Plot Orbitals Options Isosurface Rendering. Orbital Contour Value 0.05. Wire meses Isosufgace Gird. Gird Meshes size Coarse. Gird Layout Default. Gird Contour Default. Transparency Level Default.

Parámetros para el cálculo de Potencial Electrostático.

Plot Molecular Graphs. Plot Molecuar Options. Molecular Properties: Properties. Electrostatic Potential Yes. Representations 3D Mapped Isosurface. Gird Mesh Size Coarse. Gird Layout Default. Contour Gird Default. Isosurface Reading. Total Charge 
Density Contour Value 0.015. Rendering Wire Mesh. Transparency Level Default. Mapped Options Functions Default.

\subsection{Fórmulas analíticas}

$\mathrm{ETC}=\mathrm{BG} / \mathrm{EP}$

Ecuación 1

Dónde:

ETC $=$ Coeficiente de Transferencia de Electrones. Se considera adimensional. Siglas en inglés.

$\mathrm{BG}=$ Banda Prohibida. ElectrónVoltios (eV; siglas en inglés).

$\mathrm{EP}=$ Potencial Electrostático. ElectrónVoltios/ radio de Bohr $\frac{e V}{a^{0}}$ (el radio de Bohr, unidad adimensional).

$\mathrm{BG}=|\mathrm{HOMO}-\mathrm{LUMO}|$

Ecuación 2

Dónde:

HOMO = Orbital más ocupado de electrones (Highest Occupied Molecular Orbital),

LUMO = Orbital menos ocupado de electrones (Lowest Unoccupied Molecular Orbital).

$\mathrm{BG}=\mid \mathrm{E}-\mathrm{-E}+$

Ecuación 3

Dónde:

E- = Polo negativo del potencial electrostático de la molécula.

$\mathrm{E}_{+}=$Polo positivo del potencial electrostático de la molécula.

\section{RESULTADOS Y DISCUSIÓN}

\subsection{Solubilidad de AFB1 y AFM1}

En la Figura 1 se muestra que la AFB1 es una toxina de mediana solubilidad en agua. El pozo más profundo ETC= 40.533 nos indica que la interacción Agua-AFB1 es la más probable y que la AFB1 actúa preferentemente como agente oxidante (predomina el LUMO de la molécula, lado derecho de la interacción). 


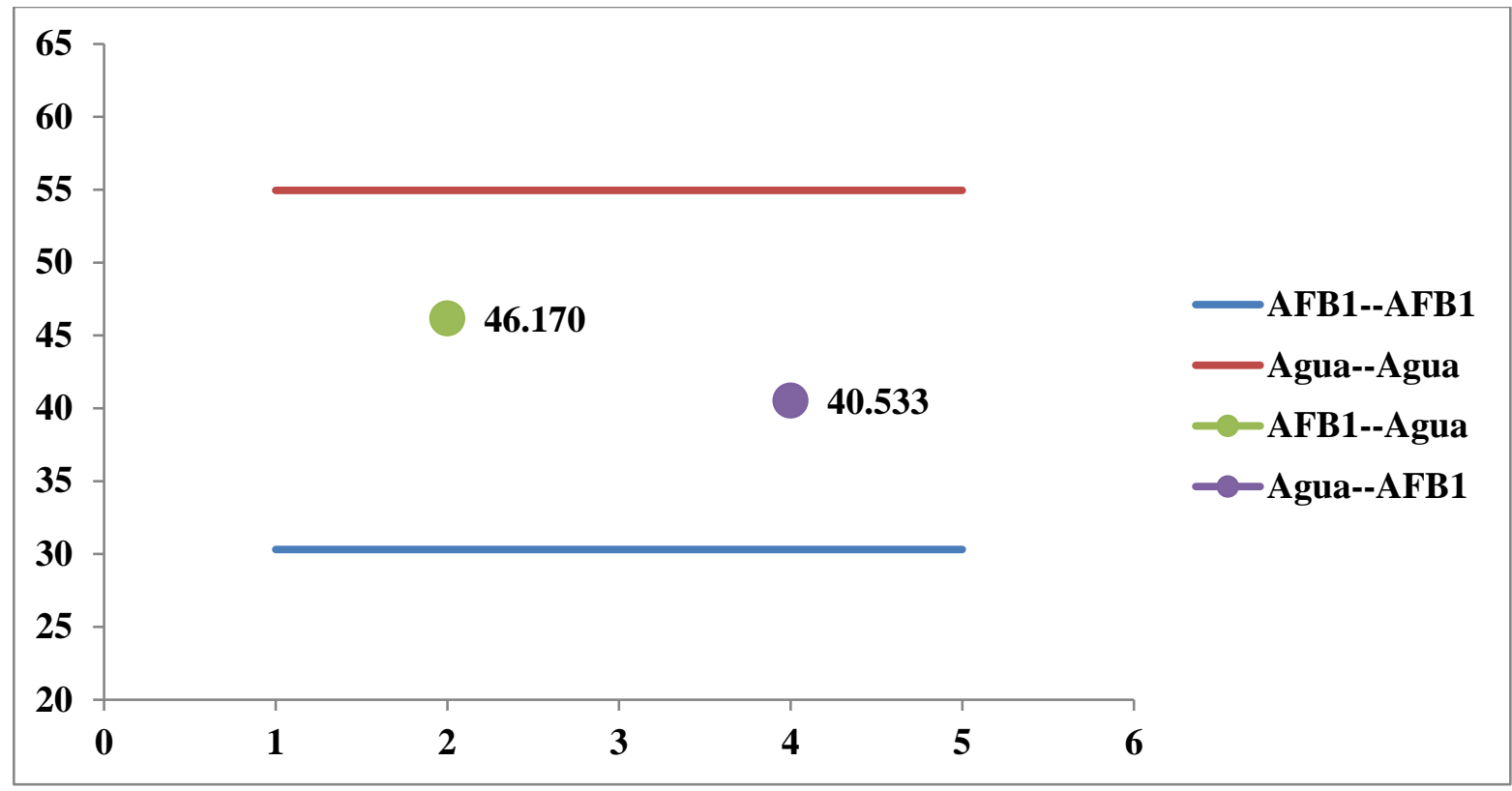

Fig. 1. Pozos cuánticos (ETCs) de la interacción del AFB1 vs Agua en la zona de probabilidad media. Esto quiere decir que la toxina AFB1 no es muy soluble en agua. El pozo más profundo (40.533) indica menor impedancia para que predomine el carácter oxidante de la AFB1 (lado derecho de la interacción).

En la figura 2 se observa la interacción del derivado metabólico AFM1. Podemos observar que su ETC = 36.023 (bajó un poco más respecto a su toxina). Este descenso nos indica una menor interacción molecular (IM) con respecto al agua, o sea, una mejor interacción de esta molécula con el agua y por lo tanto una mejor solubilidad. Tal vez esta es la primera barrera de defensa que presenta el cuerpo para excretar la toxina por la orina. Sin embargo, se mantiene el carácter oxidante de la toxina metabolizada. Este carácter oxidante es el que puede provocar mutaciones a los ácidos nucleicos.

Una mutación es el cambio de una base nitrogenada por otra en cualquiera de los ácidos nucleicos. Con este cambio la información es alterada en sus codones y por lo tanto los aminoácidos se ensamblan en orden diferente. Este nuevo orden hace que se pierda el control en cualquier parte del sistema metabólico. De esta forma se provoca un caos, un aumento entrópico que genera producción masiva no deseada de proteínas y otras sustancias vitales para el funcionamiento metabólico. 


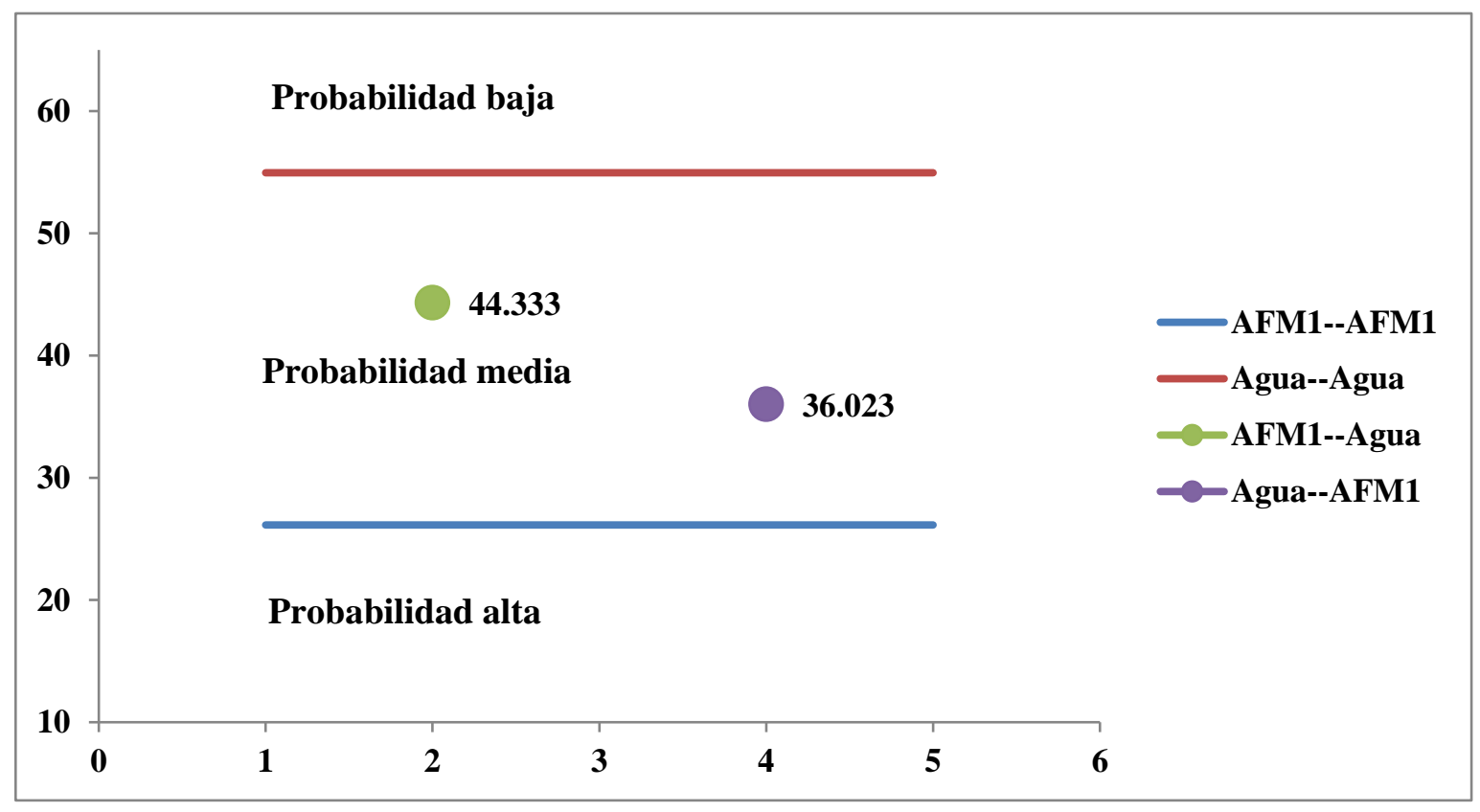

Fig. 2. Pozos cuánticos (ETCs) de la interacción del derivado metabólico AFM1 vs Agua en la zona de probabilidad media. El derivado metabólico AFM1 aumenta su solubilidad (36.023); pero continúa con su papel oxidante.

\subsection{Bandas cruzadas del AFB1, AFM1 vs pares de bases nitrogenadas permitidas}

En la Tabla 1 se muestra el valor de los ETCs de las bases para el ADN y ARN. La toxina AFB1 $(\mathrm{ETC}=30.310)$ es más inestable que las bases. Esto quiere decir que es muy agresiva y puede romper estos frágiles puentes de hidrógenos tanto del ADN como del ARN. Sin embargo su derivado metabólico la AFM1 (ETC = 26.139) es menos agresivo, debido a que su ETC es menor. Esto no asegura que aún el derivado metabólico no compita por los puentes de hidrógeno de las bases permitidas. Para detallar mejor las interacciones, se tomaron como un ente químico los pares de bases para realizar análisis de bandas cruzadas de los pares permitidos vs la AFB1 y el AFM1. Los resultados se muestran en la Tabla 2.

Se puede observar con sintonía cuántica más fina los resultados de la Tabla 2. Se puede ver que contrario a lo que se afirmaba en base a los resultados de la Tabla 1, el derivado metabólico es mucho más agresor que la misma toxina si compiten entre ellos mismos; juntos tienen una alta probabilidad de causar severas mutaciones tanto al ADN como al ARN (las diferencias entre sus ETCs son pequeñas). Con más detalle, el par más vulnerable por el AFM1 es C:G; en cambio el par más vulnerable para la AFB1 es A:T. Por lo tanto, ambos tienen como blanco principal al ADN. 
Tabla 1. Interacción de las bandas cruzadas entre las bases permitidas.

\begin{tabular}{lllllllll}
\hline Reductor & Oxidante & HOMO & LUMO & BG & E- & E+ & EP & ETC \\
\hline AFB1 & AFB1 & -9.283 & -1.372 & 7.911 & -0.118 & 0.143 & 0.261 & 30.310 \\
A & T & -8.654 & -0.475 & 8.179 & -0.140 & 0.169 & 0.309 & 26.469 \\
A & U1* & -8.654 & -0.511 & 8.143 & -0.140 & 0.171 & 0.311 & 26.183 \\
AFM1 & AFM1 & -9.373 & -1.113 & 8.260 & -0.132 & 0.184 & 0.316 & 26.139 \\
C & G & -9.142 & -0.206 & 8.936 & -0.174 & 0.172 & 0.346 & 25.827 \\
A & U2** & -8.654 & -0.415 & 8.239 & -0.140 & 0.202 & 0.342 & 24.091 \\
\hline
\end{tabular}

Tautómeros del Uracilo: * Cetónico, **Hidroxílico

Se puede reafirmar entonces, que la deducción de muchos investigadores de que la AFB1 es una de las sustancias más cancerígenas que existe, es cierto, debido a que aún metabolizada, por lo menos con el AFM1, se vuelve más mutágenica.

En la Tabla 2 se muestran todas las posibles interacciones de la toxina AFB1 y su derivado metabólico AFM1. Con asteriscos se señalan las interacciones más probables que pueden romper los puentes de $\mathrm{H}$ de las bases nitrogenadas permitidas. Nótese que el derivado metabólico ataca con mayor precisión a la base permitida C:G. Otra de las cosas que podemos ver en esta tabla es que tanto AFB1 como AFM1 son oxidantes y jamás se van a comportar como antioxidantes (reductores) debido a su alto ETC. 
Tabla 2. Interacciones bandas cruzadas de la toxina AFB1 y su derivado metabólico AFM1 vs enlaces de $\mathrm{H}$ de las bases nitrogenadas permitidas del ADN y ARN.

\begin{tabular}{|c|c|c|c|c|c|c|c|c|c|}
\hline No. & Reductor & Oxidante & HOMO & LUMO & BG & E- & E+ & EP & ETC \\
\hline 1 & AFB1 & $C: G$ & -9.283 & -0.206 & 9.077 & -0.118 & 0.172 & 0.290 & 31.300 \\
\hline 2 & AFB1 & $\mathrm{A}: \mathrm{T}$ & -9.283 & -0.475 & 8.808 & -0.118 & 0.169 & 0.287 & 30.690 \\
\hline 3 & AFB1 & A:U1 & -9.283 & -0.511 & 8.772 & -0.118 & 0.171 & 0.289 & 30.353 \\
\hline 4 & AFB1 & AFB1 & -9.283 & -1.372 & 7.911 & -0.118 & 0.143 & 0.261 & 30.310 \\
\hline 5 & AFM1 & $C: G$ & -9.373 & -0.206 & 9.167 & -0.132 & 0.172 & 0.304 & 30.155 \\
\hline 6 & AFM1 & $\mathrm{A}: \mathrm{T}$ & -9.373 & -0.475 & 8.898 & -0.132 & 0.169 & 0.301 & 29.561 \\
\hline 7 & AFM1 & A:U1 & -9.373 & -0.511 & 8.862 & -0.132 & 0.171 & 0.303 & 29.248 \\
\hline 8 & AFM1 & AFB1 & -9.373 & -1.372 & 8.001 & -0.132 & 0.143 & 0.275 & 29.095 \\
\hline 9 & AFB1 & A:U2 & -9.283 & -0.415 & 8.868 & -0.118 & 0.202 & 0.320 & 27.713 \\
\hline 10 & A:U2 & $C: G$ & -8.654 & -0.206 & 8.448 & -0.14 & 0.172 & 0.312 & 27.077 \\
\hline 11 & A:U1 & $C: G$ & -8.654 & -0.206 & 8.448 & -0.14 & 0.172 & 0.312 & 27.077 \\
\hline 12 & $\mathrm{~A}: \mathrm{T}$ & $C: G$ & -8.654 & -0.206 & 8.448 & -0.14 & 0.172 & 0.312 & 27.077 \\
\hline 13 & AFB1 & AFM1 & -9.283 & -1.113 & 8.170 & -0.118 & 0.184 & 0.302 & 27.053 \\
\hline 14 & AFM1 & A:U2 & -9.373 & -0.415 & 8.958 & -0.132 & 0.202 & 0.334 & 26.820 \\
\hline 15 & A:U2 & $\mathrm{A}: \mathrm{T}$ & -8.654 & -0.475 & 8.179 & -0.14 & 0.169 & 0.309 & 26.469 \\
\hline 16 & A:U1 & $\mathrm{A}: \mathrm{T}$ & -8.654 & -0.475 & 8.179 & -0.14 & 0.169 & 0.309 & 26.469 \\
\hline 17 & $\mathrm{~A}: \mathrm{T}$ & $\mathrm{A}: \mathrm{T}$ & -8.654 & -0.475 & 8.179 & -0.14 & 0.169 & 0.309 & 26.469 \\
\hline 18 & A:U2 & A:U1 & -8.654 & -0.511 & 8.143 & -0.14 & 0.171 & 0.311 & 26.183 \\
\hline 19 & A:U1 & A:U1 & -8.654 & -0.511 & 8.143 & -0.14 & 0.171 & 0.311 & 26.183 \\
\hline 20 & $\mathrm{~A}: \mathrm{T}$ & A:U1 & -8.654 & -0.511 & 8.143 & -0.14 & 0.171 & 0.311 & 26.183 \\
\hline 21 & AFM1 & AFM1 & -9.373 & -1.113 & 8.260 & -0.132 & 0.184 & 0.316 & 26.139 \\
\hline 22 & $C: G$ & $C: G$ & -9.142 & -0.206 & 8.936 & -0.174 & 0.172 & 0.346 & 25.827 \\
\hline 23 & A:U2 & $\mathrm{AFB} 1 * *$ & -8.654 & -1.372 & 7.282 & -0.14 & 0.143 & 0.283 & 25.731 \\
\hline 24 & A:U1 & $\mathrm{AFB} 1 * *$ & -8.654 & -1.372 & 7.282 & -0.14 & 0.143 & 0.283 & 25.731 \\
\hline 25 & $\mathrm{~A}: \mathrm{T}$ & $\mathrm{AFB} 1 * *$ & -8.654 & -1.372 & 7.282 & -0.14 & 0.143 & 0.283 & 25.731 \\
\hline 26 & $\mathrm{C}: \mathrm{G}$ & $\mathrm{A}: \mathrm{T}$ & -9.142 & -0.475 & 8.667 & -0.174 & 0.169 & 0.343 & 25.268 \\
\hline 27 & $C: G$ & A:U1 & -9.142 & -0.511 & 8.631 & -0.174 & 0.171 & 0.345 & 25.017 \\
\hline 28 & $C: G$ & $\mathrm{AFB} 1 * *$ & -9.142 & -1.372 & 7.770 & -0.174 & 0.143 & 0.317 & 24.511 \\
\hline 29 & A:U2 & A:U2 & -8.654 & -0.415 & 8.239 & -0.14 & 0.202 & 0.342 & 24.091 \\
\hline 30 & A:U1 & A:U2 & -8.654 & -0.415 & 8.239 & -0.14 & 0.202 & 0.342 & 24.091 \\
\hline 31 & $\mathrm{~A}: \mathrm{T}$ & A:U2 & -8.654 & -0.415 & 8.239 & -0.14 & 0.202 & 0.342 & 24.091 \\
\hline 32 & A:U2 & AFM1* & -8.654 & -1.113 & 7.541 & -0.14 & 0.184 & 0.324 & 23.275 \\
\hline 33 & A:U1 & AFM1* & -8.654 & -1.113 & 7.541 & -0.14 & 0.184 & 0.324 & 23.275 \\
\hline 34 & $\mathrm{~A}: \mathrm{T}$ & AFM1* & -8.654 & -1.113 & 7.541 & -0.14 & 0.184 & 0.324 & 23.275 \\
\hline 35 & $\mathrm{C}: \mathrm{G}$ & A:U2 & -9.142 & -0.415 & 8.727 & -0.174 & 0.202 & 0.376 & 23.210 \\
\hline 36 & $\mathrm{C}: \mathrm{G}$ & AFM1* & -9.142 & -1.113 & 8.029 & -0.174 & 0.184 & 0.358 & 22.427 \\
\hline
\end{tabular}

*Interacciones muy probables para romper puente de hidrógeno de las bases permitidas.

**Interacciones probables para para romper puente de hidrógeno de las bases permitidas. 


\subsection{Ilustración de casos específicos de pozos cuánticos}

En esta sección se ilustrarán los casos más sobresalientes usando los pozos cuánticos. En las figuras, los puntos representan el fondo de cada pozo cuántico de las interacciones cruzadas de dos entes químicos dados interactuantes; tal y como se mencionó anteriormente. Las líneas son los límites (pozos cuánticos o ETCs) de esos dos entes químicos. Entre más profundo sea el pozo cuántico existe más probabilidad de que se dé la interacción. La IM entre más baja sea, menos resistencia encuentra el electrón para pasar de una molécula a otra y esto trae como consecuencia desde una corriente eléctrica hasta una reacción química.

Observamos cuatro de los casos más relevantes de estas interacciones (Figs. 3-6). El punto más bajo indica una menor IM para el salto cuántico del electrón de una molécula a otra. El punto más alto indica una mayor IM. La zona debajo de la línea más baja indica que la IM es más baja inclusive que la IM de los dos entes químicos en cuestión y en su estado puro. Por lo tanto esta zona es la de mayor probabilidad de que ocurra la transferencia o salto cuántico y de esta manera haya un fenómeno físico o químico con esas dos moléculas. En este caso una mutación.

Por otro lado, en las tablas 1 y 2 se observa que las moléculas colocadas del lado izquierdo tienen un carácter reductor, mientras que las moléculas colocadas del lado derecho, tienen un carácter oxidante. Esto se debe que al lado izquierdo se colocaron los HOMOs y al lado derecho los LUMOs y sus respectivos potenciales. Los electrones siempre saltan de HOMO en dirección de LUMO.

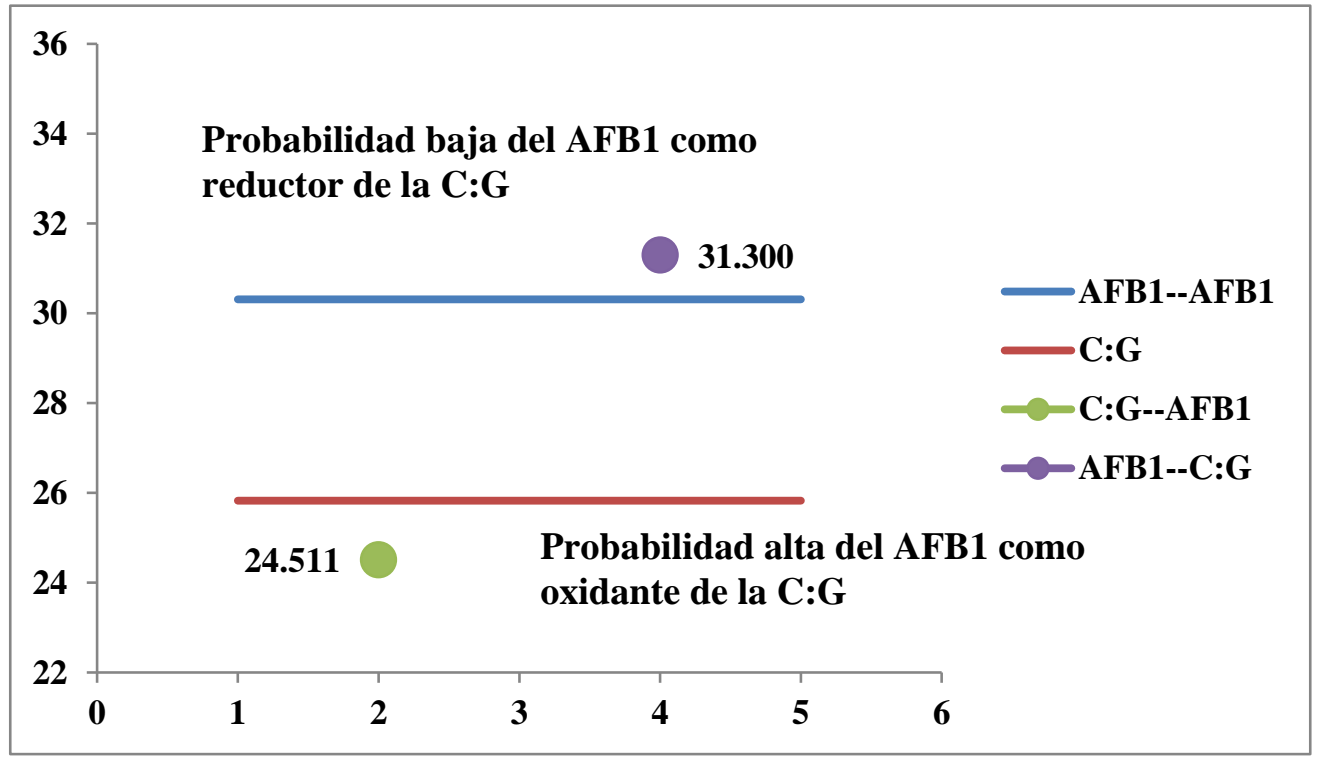

Fig. 3. Pozo cuántico del ataque de la AFB1 al par de bases C:G. Se observa que el pozo más profundo (color verde) es cuando la AFB1 ataca al par de pases C:G como agente oxidante. 


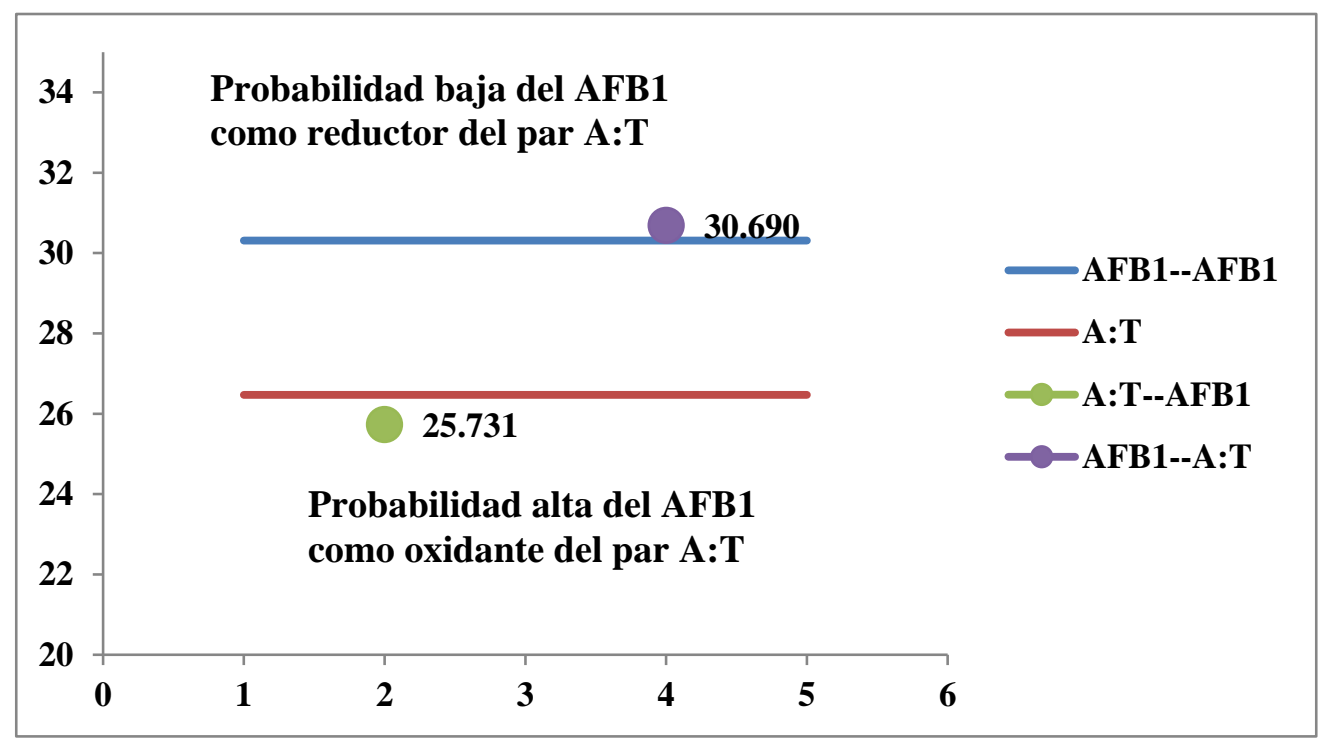

Fig. 4. Pozo cuántico del ataque de la AFB1 al par A:T. Se observa un patrón similar al ataque de la C:G.

En todas las figuras se observan dos cosas que determinan el carácter oxidativo de la AFB1: 1) En todos ellos aparece la AFB1 de lado derecho y coincide con... 2) El punto más bajo o sea el pozo cuántico más profundo (verde).



Fig. 5. Pozo cuántico del ataque de la AFB1 al par C:U2. Nótese que el ataque al ARN es de probabilidad media y sigue el mismo patrón, el AFB1 es agente oxidante. 


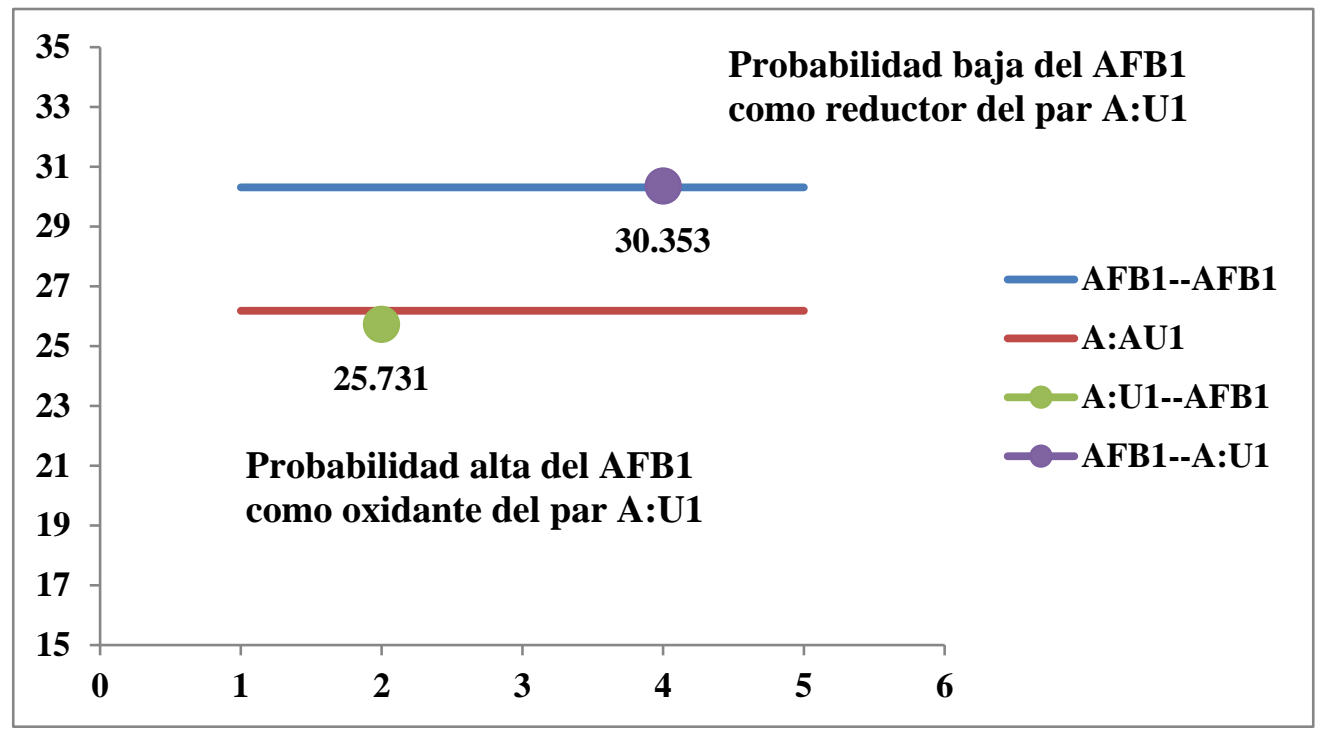

Fig. 6. Pozo cuántico del ataque de la AFB1 al par de bases C:U1. En este ataque el AFB1 continúa su labor como agente oxidante en una zona de probabilidad alta.

En las figuras 4-10 se presentan los casos más significativos del derivado metabólico AFM1. Se observa un patrón casi exacto, con una diferencia pequeña en cuanto a los valores de los ETCs de las interacciones con los mismos pares de bases permitidos. Eso significa que aunque el cuerpo trate de eliminar la toxina AFB1 convirtiéndola en el derivado metabólico AFM1 aumentando su solubilidad; su peligrosidad no solo se conserva sino que aumenta.

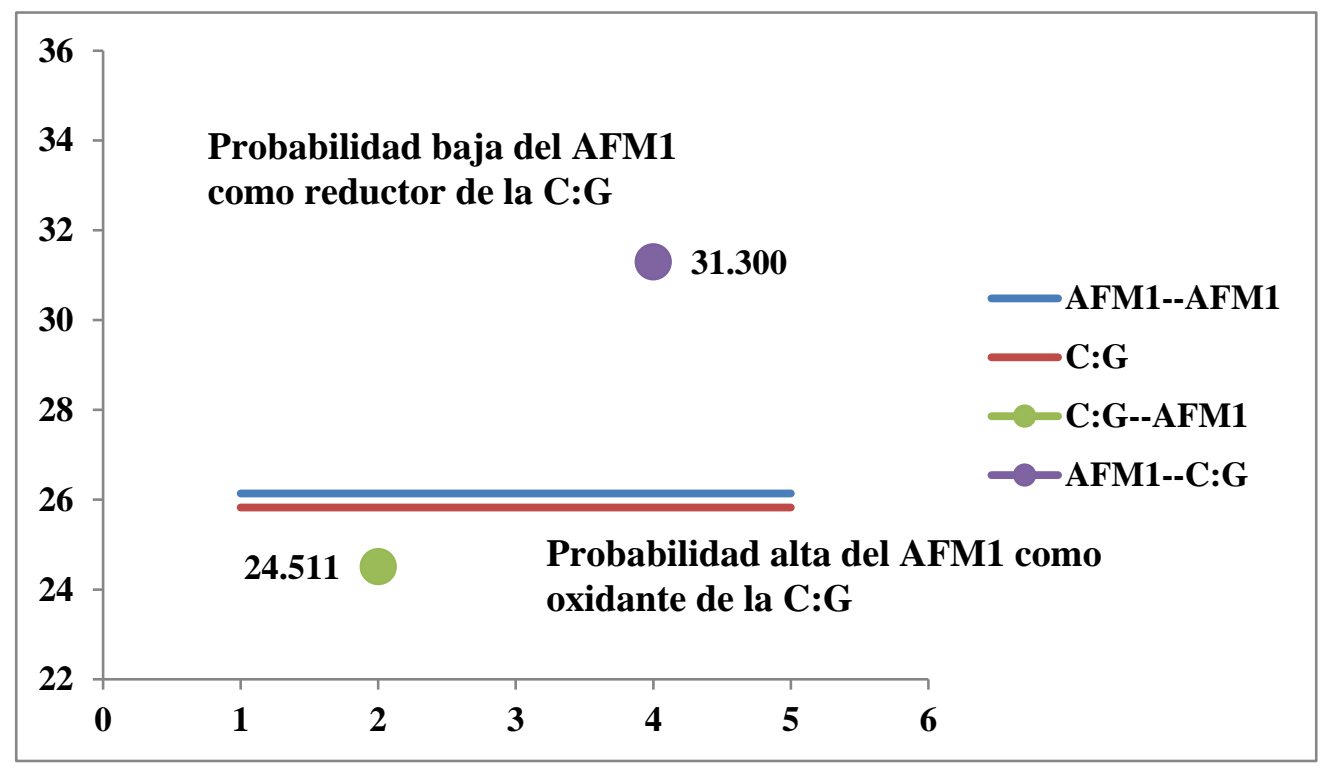

Fig. 7. Pozo cuántico del ataque de la AFM1 al par de bases C:G. El punto verde indica el pozo cuántico más probable para que el AFM1 oxide al par de bases C:G. Inclusive es mucho menor que el pozo cuántico del ataque del AFB1 al mismo par de bases . 


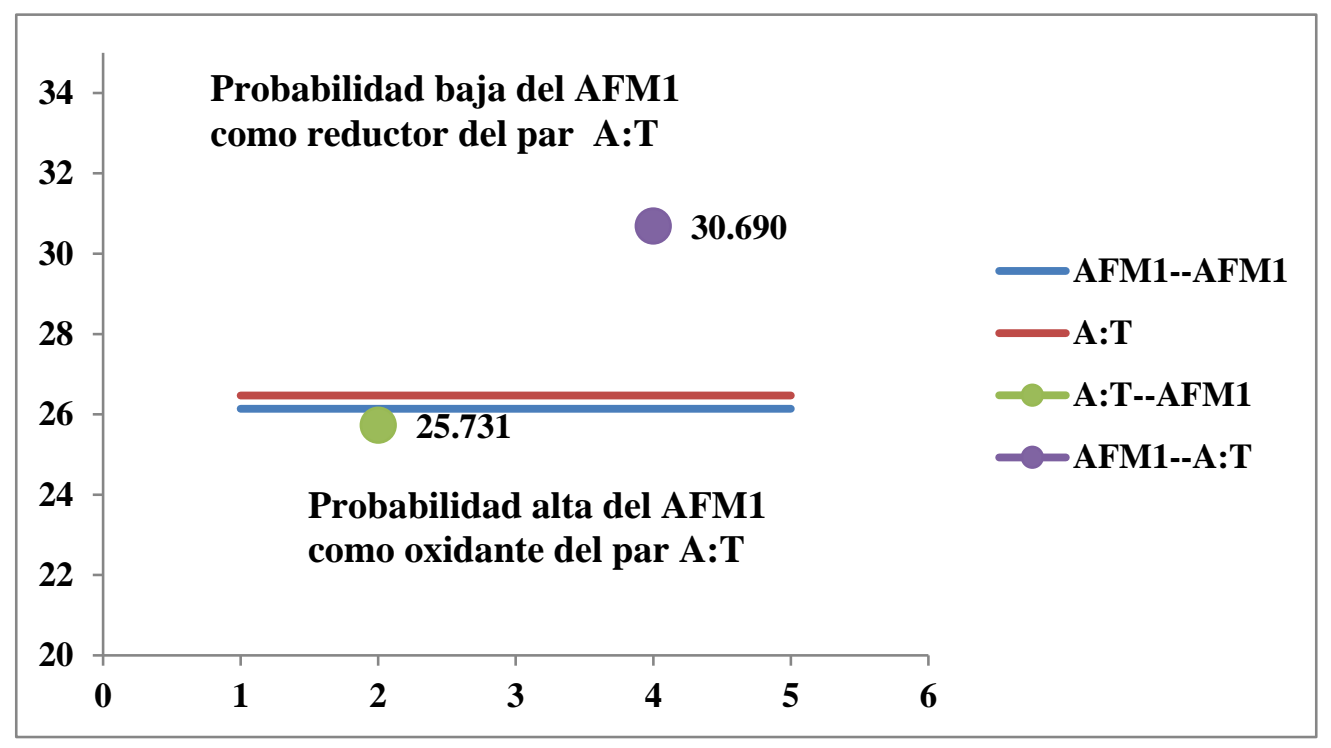

Fig. 8. Pozo cuántico del ataque de la AFM1 al par de bases A:T. En este ataque se ve que el pozo cuántico más bajo (verde) tiene el mismo valor y el mismo patrón que el ataque AFB1 al par de bases A:T.

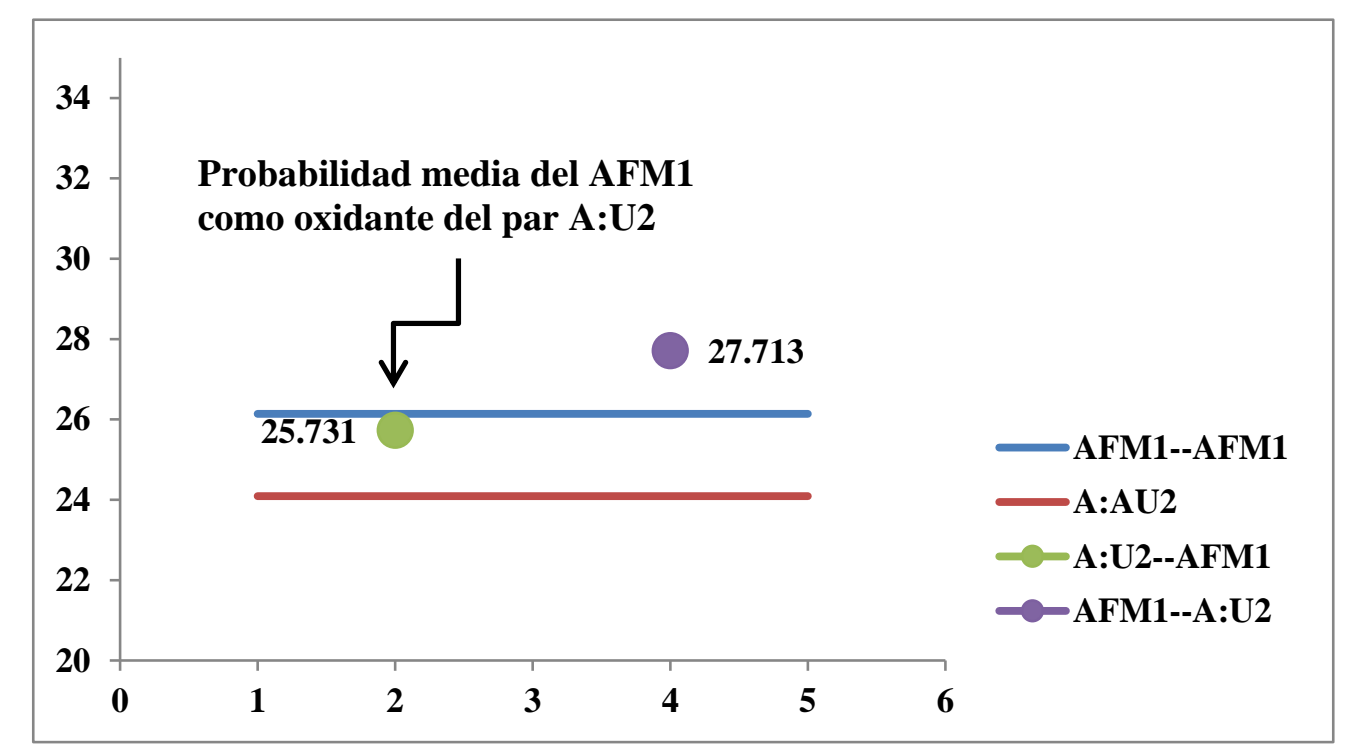

Fig. 9. Pozo cuántico del ataque de la AFM1 al par de bases A:U2. 


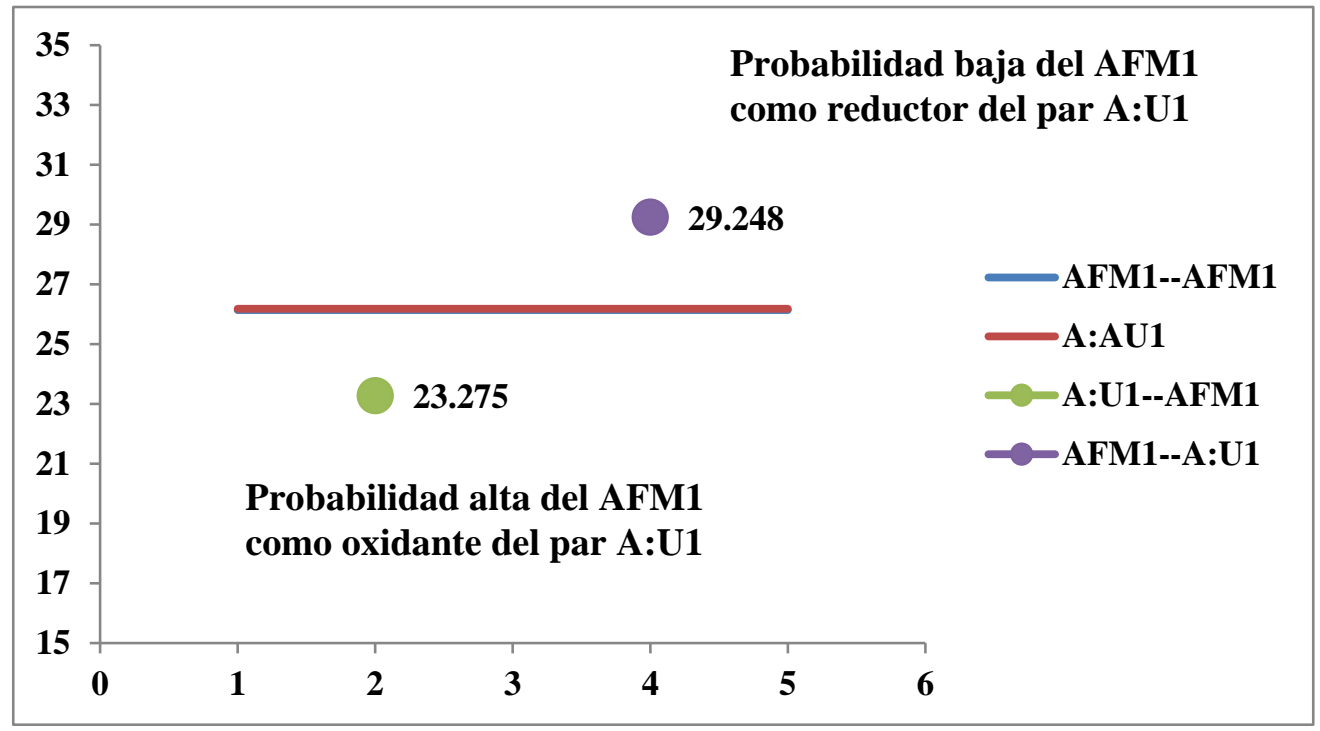

Fig. 10. Pozo cuántico del ataque de la AFM1 al par de bases A:U1

Estas diferencias se muestran con asteriscos en la Tabla 2.

\section{CONCLUSIONES}

En relación a la solubilidad se observa que el sistema biológico trata de eliminar la toxina AFB1 $($ ETC $=40.533)$ a través de su derivado metabólico AFM1 $(\mathrm{ETC}=36.023)$ que es más soluble. Se concluye que la solubilidad se debe a la disminución del ETC (impedancia molecular) por banda cruzada con el agua. En lo que respecta a las bandas cruzadas se encontró una alta probabilidad mutagénica tanto para AFB1 como para AFM1. AFM1 es más mutagénica que AFB1. En relación al ETC se encontró que las dos moléculas se comportan como agentes oxidantes debido a que son moléculas que absorben electrones. Este hallazgo se muestra debido a la profundidad de los pozos cuánticos; entre más profundo es un pozo cuántico mayor probabilidad tiene de que los electrones sean absorbidos por AFB1 y AFM1. La toxina AFB1 tiene un muy alto poder mutagénico, pero su derivado metabólico AFM1 tiene una capacidad mutagénica aún mayor. Esto quiere decir que el cuerpo humano al tratar de eliminar la toxina (AFB1), la transforma metabólicamente a una sustancia altamente cancerígena. 


\section{REFERENCIAS}

Awuor, A. O., Yard, E., Daniel, J. H., Martin, C., Bii, C., Romoser, A., \& Zitomer, N. C. 2017. Evaluation of the efficacy, acceptability and palatability of calcium montmorillonite clay used to reduce aflatoxin B1 dietary exposure in a crossover study in Kenya. Food Additives \& Contaminants: Part A, 34(1), 93-102.

Bilandžić, N., Varenina, I., Solomun Kolanović, B., Božić Luburić, Đ., Varga, I., Želježić, B. \& Cvetnić, Ž. 2017. Occurrence of aflatoxin M1 in raw cow, goat and sheep milk during spring and autumn in Croatia during 2016. Toxin Reviews, 1-7.

Chawanthayatham, S., Valentine, C. C., Fedeles, B. I., Fox, E. J., Loeb, L. A., Levine, S. S. \& Essigmann, J. M. 2017. Mutational spectra of aflatoxin B1 in vivo establish biomarkers of exposure for human hepatocellular carcinoma. Proceedings of the National Academy of Sciences, 201700759.

Chu, Y. J., Yang, H. I., Wu, H. C., Liu, J., Wang, L. Y., Lu, S. N. \& Chen, C. J. 2017. Aflatoxin B1 exposure increases the risk of cirrhosis and hepatocellular carcinoma in chronic hepatitis B virus carriers. International Journal of Cancer. 141(4):711-720.

De Guadalupe Moctezuma-Zarate, M., Carvajal-Moreno, M., Espinosa-Aguirre, J. J., Gonsebatt-Bonaparte, M. E., Rojo-Callejas, F., Perez-Lopez, P. C. U. I. \& Ruiz-Velasco, S. 2015. Role of $\mathrm{pH}$ in the mutagenicity of aflatoxin B1 in maize tortillas during in vitro human digestion model. Journal of Food and Nutritional Disorders. 4:3

Fedeles, B. I., Chawanthayatham, S., Croy, R. G., Wogan, G. N., \& Essigmann, J. M. 2017. Early detection of the aflatoxin B1 mutational fingerprint: a diagnostic tool for liver cancer. Molecular \& Cellular Oncology, (just-accepted), 00-00.

González-Pérez, M., Briteño-Vázquez, M., García-Barrera, F. A., Ham-Tirado, A. K., López-Oglesby, J. M., Salazar-Amador, M. R., \& Pacheco-García, P. F. 2016. Molecular interactions of nicotine and the nitrogenous bases of DNA and RNA calculated by quantum methods. World Journal of Pharmaceutical Research, 5(3), 1778-1792.

González-Pérez, M., Pacheco-Bautista, D., Ramirez-Reyes-Montaño, H. A., Medel-Rojas, A., González-Murueta, J. W. \& Sánchez, C. 2017. Analysis of the interactions of n-(1- $\alpha-$ aspartil)-1-phenylalanine, 1-metil ester (aspartame) and the nitrogen bases of DNA and RNA using quantum methods. World Journal of Pharmaceutical Research, 6(5), 40-49.

Jafari, T., Fallah, A. A., Kheiri, S., Fadaei, A., \& Amini, S. A. 2017. Aflatoxin M1 in human breast milk in Shahrekord, Iran and association with dietary factors. Food Additives \& Contaminants: Part B, 10(2), 128-136.

Kim, S., Lee, H., Lee, S., Lee, J., Ha, J., Choi, Y. \& Choi, K. H. 2017. Invited review: Microbe-mediated aflatoxin decontamination of dairy products and feeds. Journal of dairy science, 100(2), 871-880. 
Livingstone, M. C., Johnson, N. M., Roebuck, B. D., Kensler, T. W., \& Groopman, J. D. 2017. Profound changes in miRNA expression during cancer initiation by aflatoxin B1 and their abrogation by the chemopreventive triterpenoid CDDO-Im. Molecular Carcinogenesis. doi: 10.1002/mc.22635.

Mary, V. S., Arias, S. L., Otaiza, S. N., Velez, P. A., Rubinstein, H. R. \& Theumer, M. G. 2017. The aflatoxin B1-fumonisin B1 toxicity in BRL-3A hepatocytes is associated to induction of cytochrome P450 activity and arachidonic acid metabolism. Environmental Toxicology. 32(6):1711-1724.

Miocinovic, J., Keskic, T., Miloradovic, Z., Kos, A., Tomasevic, I. \& Pudja, P. 2017. The aflatoxin M1 crisis in the Serbian dairy sector: the year after. Food Additives \& Contaminants: Part B, 10(1), 1-4.

Olsen, L., Jørgensen, F. S. \& Bonomo, S. 2017. Dissecting the cytochrome P450 1A2 and $3 \mathrm{~A} 4$ mediated metabolism of aflatoxin B1 in ligand and protein contributions. Chemistry-A European Journal, 23(12), 2884-2893

Schwartzbord, J., Severe, L. \& Brown, D. 2017. Detection of trace aflatoxin M1 in human urine using a commercial ELISA followed by HPLC. Biomarkers, 22(1), 1-4. 